\title{
Applications of Virtual Reality in Mental Health
}

\author{
Malvika Singh, Kaveri Devi Mishra
}

\begin{abstract}
This objective of this research is to study the application of virtual reality in health care and to especially comprehend its role in mental health care. The methodology employed in qualitative in nature, mainly descriptive that takes in to consideration the databases of various journals and academic websites. The research paper aims at providing a review and analysis on how virtual reality or the artificial environment is used to treat various health related issues, paying specific attention on the mental health and related ailments. Virtual reality has taken a great leap in the past two decades where its various applications are being used to not only treat different medical conditions but also provide training to the medical professionals, thereby reducing the cost of training manifold. The use of artificial environment has improved the quality of health care in the recent years. However, there are still few challenges that the virtual reality world faces and needs to address. The applications of virtual reality need to be communicated widely as to increase the awareness among people and lessen the stigma attached with getting help for mental health issues.
\end{abstract}

Keywords: Virtual reality, computer, technology, applications, health care, mental health.

\section{INTRODUCTION:}

According to the World Health Organization website, more than 20 million people worldwide suffer from schizophrenia and more than 300 million people with other forms of mental disorders

(www.who.int/en/news-room/fact-sheets/detail/mental-disor ders). Virtual reality is a term used for artificial environment in which the user experiences immersive or non-immersive techniques to enter into alternate realities and interact with immerses himself in the computer simulated artificial reality varies (Weimer, 1994). Virtual Reality as a technology came into being in 1950s but it was only after 1980s and 1990s that this technology gained public notice. The credit of this goes to Jaron Lanier who coined term virtual reality in 1987. The other terms used to define the virtual environment are Artificial Reality (Krueger, 1991) and Cyberspace (Gibson, 1984). The term Virtual worlds has also been used in recent times to explain the alternate reality. This technology aids people to see and experience things that are not accessible in real life. It can also be manipulated and is only limited by imagination. It also allows users to not only interact with the interface but also participate in it. There are various tools that enable a user to interact and participate in the virtual environment. These tools help in making the experience as closer to reality as possible mainly through the sense of vision and auditory. However, to make the experience even more

Revised Version Manuscript Received on 16 September, 2019.

* Correspondence Author

*Malvika Singh - M.A. (Mass Communication and Journalism), JRF, PhD Scholar, Amity School of Communication, Amity University, Noida

*Dr. Kaveri Devi Mishra - Associate Professor, Amity School of Communication, Amity University, Noida them (Van Dam et al, 2000). The degree to which the user

realistic, the senses of smell and touch are also being taken care of. The presence of various tools (Mandal,2013) makes the virtual experience closer to the reality as much as possible. Some of them are-

- Morton Heilig created a multi-sensory simulator called Sensorama in 1957 and got it patented in 1962. This machine had the elements of sight, smell, wind and vibration but the only limitation was that it was not interactive like the modern virtual reality machines.

- In 1965, one of the pioneers in the field of virtual reality, Ivan Sutherland came up with the concept of an ultimate escape with the elements of sight, smell, touch and taste along with force feedback and interactive graphics.

- Ivan Sutherland is also credited with creating the first HMD (Head Mounted Display) which adjusted itself according to the user's head position and orientation.

- In 1971, the University of Carolina came up with the first prototype of a force-feedback system called GROPE.

- VIDEOPLACE was created as a 2D technology which integrated the computer generated graphics with the actions performed by the user.

- In 1982, the Visually Coupled Airborne System Simulator (VCASS) was introduced by the United States' air force's Armstrong Medical Research Laboratories to simulate flights. This tool was designed to provide training to the pilots in which they had to wear a Head Mounted Display that guided them on targeting and navigation.

- In 1984, the VIrtual Visual Environment Display (VIVED) was developed by the National Aeronautics and Space Administration (NASA).

- In 1985 and 1988 respectively, the most polular virtual reality devices DataGlove and Eyephone HMD were introduced.

- BOOM came into existence much earlier was commercialized in 1989. This device has two eye holes through which the user peeps and operates the mechanic arms attached.

- The Virtual Wind Tunnel was again created by the National Aeronautics and Space Administration (NASA) in 1990s with the help of already existing technologies of DataGlove and Boom.

- In 1992, the Cave Automatic Virtual Environment (CAVE) came into existence. This device doesn't need the user to wear a Head Mounted Display but only an LCD shutter glass that enables the user to see the stereoscopic images on the wall. This device aids in superior quality images. 
Virtual reality scores more than the mainstream media because of its three dimensional nature. It has interactivity and participation as its main features. There are various levels of immersions in virtual reality systems - Non-immersive, Semi-immersive and Immersive (Mandal, 2013).

Non-Immersive - Also called the desktop virtual reality, this is the lowest form of VR in which no fancy devices are needed. In this, one or more screens are connected to computer. The monitor usually displays the images only and no other sensory output is provided. The user can utilize but not interact with this system.

Semi-immersive - Also called the fish tank VR system, this system is a refined version of the desktop VR system. In this system also, a monitor is used but with shutter glasses so that more realistic visuals are received by the user.

Immersive - This system includes the Head Mounted Display and other tools to aid in making the simulation as closer to reality as possible. Almost all the senses are employed such as vision, auditory, touch and smell in order to make the user experience the reality as closely as possible.

The virtual environment not only helps in simulating the reality but also helps experience situations that do not exist. Virtual reality has found its applications in various fields including medicine, engineering, education, construction, car design, biology etc.

In this paper, we will be focusing on health care paying specific attention on mental health. The applications of virtual reality in health care have increased in the past decade and have helped in saving on the cost to a great length. These have become involved in but not limited to surgical procedures, surgical simulations, training of medical professionals, treatment of mental health issues such as phobias and other psychological disorders, medical education, patient education etc. Tele-presence or remote surgery is a technique in which a surgeon performs a surgery by manipulating the robotics, sitting at a remote location. This tool can help save the lives of soldiers and general people altogether when a surgeon is not available. This can be used in the service of armed forces as well as in the remote locations or rural areas. Another example can be endoscopy in which a micro camera is sent inside the body of the patient to detect exactly where the disease is. Virtual reality can also be used for minimally invasive surgery in which computer generated graphics are superimposed on the real images thereby providing a holistic view of the organ concerned. Here, a surgeon performs the 'operation' by manipulating the machines and a robotic 'arm' performs the same procedure on to the patient. This technique can specifically be used in neurological surgery where intricacies of brain are involved. Patients who suffer from stroke can regain substantial amount of function recovery after being exposed to the virtual reality devices. These devices activate the specific muscular activity that aids in gaining the cerebral muscle strength. In treating patients with dental problems, dentists make them wear the Head Mounted Display or 3D glasses to play audio-visual clips. This helps them distract from the pain. Same goes in the treatment of cancer patients where the Psycho-oncological VR Therapy (POVRT) application (Riva, 1997) helps them relax as they tend to get anxious in the anticipation of the pain. The virtual environment is also employed in video games to educate the patients. For example, Health Hero is a video game that has been developed for pediatric patient education. Medical institutions are coming up with simulators to provide training to the medical professionals. These include both surgical and non-surgical procedures. This helps in training the doctors and medical professionals more efficiently as cadavers are hard to get and since they are lifeless, it cannot possibly help them to see what happens when a pulsating artery is dissected. But with an artificial cadaver equipped with virtual reality system, the medical professionals can practice as they would have on a live human body.

For children who are disabled and are learning to use a wheelchair, the virtual environment will enable them to practice using the wheelchair without potential safety problems, while boosting their motor skills.

\section{METHODS:}

The approach used in this study is qualitative in nature and is based on methodical and careful analysis of secondary data. The data has been gathered through various data collection tools like research papers, journals and academic websites. The method that has been used is the review of literature method, from 1980s to 2018. The researches that included the study of virtual reality and its applications in health care and especially mental health were taken into consideration. The descriptive method through the review of literature has been adopted by various analysts in exploring different aspects of the main study. The study explores the data gathered through various sources to reach on a conclusion. There is an in depth analysis and discussion that the researcher reached to after systematic analysis of the secondary data. The methodical analysis revealed that virtual reality has played a pivotal role in the field of health care in the past two decades. However, its application in the field of mental health is still in the phase of development. The researcher has underlined the orderly analysis of the information gathered and drawn conclusion on the basis of careful study and full understanding of the available data. The limitations of this study were that the researcher had to rely on the secondary sources as collecting primary data on the topic of study is hard to achieve. The collection of primary data on virtual reality and mental health includes consulting mental health patients which is difficult because of the social stigma attached. The topic of research combines health communication and technology.

\section{VIRTUAL REALITY AND MENTAL HEALTH:}

Virtual reality has come a long way in treating mental health issues. In this paper the attention has been on phobias and post-traumatic stress disorders (PTSD). Our day to day actions are governed by our mental health and conditioning. Virtual environment helps in not only treating the mental disorders safely but also aids in getting to the base of the problem or detection of the cause. Post-traumatic stress disorder is caused when a person survives accident or a bad incident such as war or death of a family or friend. The effects of post-traumatic stress disorder can include kneejerk reaction, hallucination, anxiety, fear etc. The virtual environment can help such people by recreating the environment that makes them anxious. This will help the affected people become more and more immune to the anxiety and hallucinations they experience. After the attack of $9 / 11$, a study was conducted and the results found out that 
majority of the sufferers in the study experienced considerable improvement in their post-traumatic stress (Difede and Hoffman, 2002). A phobia is fear of something or someone to the extent that it starts hampering the normal day to day functioning of the sufferer. Phobia can be of anything, such as fear of height, fear of flying, fear of reptiles, fear of closed space etc. In such cases also, virtual reality devices can be used to recreate the environment which induces fear in the minds of the sufferer. This helps them become at ease with the stimulants that trigger the fear psychosis. Virtual reality helps overcome the phobia by creating artificial environment and thereby reducing the chance of harm that could have occurred had the sufferers been exposed to the real stimulants (Roy, 2003). The 'Emotional Processing Theory' (Hodges et al. 2001) explains exactly how sufferers are exposed to the fear inducing stimulants and learn to deal with them as reasonably as possible. Virtual reality combines artificial environment with the reality without being completely immersed (Haniff et al., 2000). The technique has also been successful in treating the fear of reptiles such as spiders. An experiment conducted by Bouchard et al. (2006) revealed that upon exposure to virtual simulation of the presence of spiders, the sufferers of phobia for spiders experienced lesser level of fear in comparison to their past. In other mental issues such as schizophrenia also, virtual environment helps in early detection and management. Schizophrenia is a mental disorder in which the sufferer hallucinates or sees and hears things that do not exist. By putting them in the virtual environment, it can easily be detected as to whether they see what they have been shown or do they see and hear things that were not the part of the virtual environment.

Virtual environment aids in combating and to some extent curing the mental disorders. Most importantly it helps the sufferers come to terms with their situation in a better manner without putting too much pressure on them that they could have experienced had they been treated in a conventional way.

\section{REVIEW OF LITERATURE AND INTERPRETATION:}

Giraldi et al in their paper 'Introduction to Virtual Reality' have talked about the virtual world and its components. This paper delves into the depths of virtual world and gives a walk through as to what exactly is virtual reality. The explanation to stereographic projection has been given. There are two types of depth perception cues - monocular and binocular in which one eye or both the eyes are used. The monocular cues produce weak perceptions whereas the binocular cues produce very strong $3 \mathrm{D}$ depth perceptions as both the eyes produce single image each that combine to become one. This paper also tells about various VR and tracking devices such as the pointer, keyboard, cyber puck, electromagnetic tracking devices etc. Also, it talks about various applications of virtual reality such as entertainment, simulation and training, engineering, medicine, archeology and arts, architecture walkthrough and scientific data analysis.

Mandal (2013) in her paper 'Brief Introduction of Virtual Reality \& its Challenges' reveals what virtual reality is and what are the various challenges faced by it. According to the paper virtual reality is a medium of creating our own virtual world which is a simulation of the reality. It's more like customizing our own reality as per our own convenience and need. This paper gives a historic overview of virtual environment and what problems lay in the route. It gives an insight into how the virtual reality came into being in 1965 by Ivan Sutherland in the form of The Ultimate Display. Before this in 1962, Sensorama came into being courtesy Morton Heilig. Also, the terminology related to virtual reality has been extensively talked about and the varying degrees of immersion are presented carefully viz non-immersive, semi-immersive and immersive VR systems. Mandal (2013) also talks about various advantages and disadvantages of the virtual world. Advantages being its application in various health care arenas such as treatment of phobias and post-traumatic stress disorder. It also can help in the training of the medical professionals which can help cost saving tremendously. The disadvantages being excessive use of virtual reality can damage not only the eyesight but can also hamper the depth perspective in the real world. Also, the excessive use of VR systems in entertainment such as online gaming can cause the users turn into sociopaths. For example, addiction of sorts has been reported an online game PUBG players. Few challenges that the paper talks about are making virtual spaces in relatively lesser time and developing better tracking systems. On an average, it takes more than a year for a group of developers to create the replica of a real setting into a virtual environment.

Molin (1997) in the paper titled 'Virtual Reality for Health Care: a survey' has extensively talked about the applications of virtual reality in the health care. This includes surgical and non-surgical procedures including remote surgery and simulating the procedure before the surgery, patient education, medical professional training and education, skill enhancement etc. The presence of remote surgery can help on the war torn regions where soldiers fight and get injured. In such cases even if there is no surgeon is present to perform the immediate surgery, the remote location surgery is possible where a surgeon manipulates the robotic arm sitting on a remote location and the machine performs the surgery simulating the actions of the surgeon. This can help in saving the lives of the brave soldiers fighting for the nation. This can also be of tremendous help in the rural areas where a specialist is not available most of the times and the patient has to be physically transported to a place where the surgeon is available and the surgery can be performed. In such cases, the virtual setup can simulate the surgery that is carried out by a surgeon in a different location. In the case of dental patients, the dentist can set them up with a Head Mounted Display or 3D glasses playing audio-visual clips. This arrangement will help them distract and they will feel the pain a lot less. For skill enhancement also, the virtual reality techniques can help great deal. For example, a disabled person who is learning to use the wheelchair can learn to use it without potential safety problems. For architectural designs also, the virtual environment can be used to come up with the design of advanced medical facilities.

Haniff et al (2014) in the paper titled 'Virtual environments for mental health issues: A review' revealed how virtual reality helps in treating various mental health issues including post-traumatic stress disorder and phobias. This paper also paid attention towards issues such as addiction and autism. According to the authors, the virtual environment helps recreate the trauma and stimulants that trigger phobias 
which helps the sufferers become immune to the stress and the fear. Also, this paper suggests that the devices of virtual environment can be used in recognizing schizophrenia at early stages. Haniff et al (2014) also suggested that the reason why the virtual environment has been successful is that it helps simulate the real environment and monitor the activities and interaction of the users.

Riva et al (2004) in the paper titled 'Virtual Reality and Psychotherapy' also focused on the advantages of using virtual environment in the field of treatment of psychological disorders. According to the authors through virtual reality, multiple scenarios, situations, unforeseen events and consequences can be practices as trial and error without the damage of the errors. It can also be used for learning, practice and testing. In using the virtual environment for psychotherapy, an environment best suited for the patients can be created in which their interaction and progress can be monitored. Other than the phobias and post-traumatic stress disorders, the VR systems can be used for the successful treatment of social phobia and eating disorders. Riva et al (2004) presented a treatment that modifies the body image issues being faced by the patients of eating disorders that include anorexia and bulimia. They suggest the use of VEBIM (Virtual Environments for Body Image Modification), in which the combination of cognitive behavioral therapy and visual-motor therapy is used to combat the disorders.

Freeman et al (2017) in the paper titled 'Virtual reality in the assessment, understanding, and treatment of mental health disorders' also discuss extensively about the involvement of the virtual reality in the treatment of mental health issues. According to the authors, mental health disorders are inseparable from the environment and by repeated exposure to the computer generated artificial environments, the patients can learn over the period of time how to handle and overcome problems. The authors suggested that VR is often misused term and is used also for the non-immersive technologies. The term should extensively be used only for the immersive technologies. Also the authors point out that despite having the potential to be used in the assessment and remedy of mental disorders, the virtual reality system has not been utilized to its optimum potential and the authentic tests for the reliability and validity have also been very few.

Huang and Alessi (1998), in their paper titled 'Current Limitation into the Application of Virtual Reality to Mental Health Research' talk mainly about the limitations of the virtual environment. The authors agree that virtual reality has the potential in the treatment of the mental disorders but they also state that the VR system is marred by technical factors. These technical factors include high costs and complexity in the creation of the virtual world simulating the real one. The virtual environment works with the help of $3 \mathrm{D}$ software which is difficult to learn. Other than this, each and every aspect of the real world needs to be constructed in $3 \mathrm{D}$ environment which takes a lot of skill and time. Even in the presence of experts, the creation of 3D environment takes up time which in turn makes it costly. These technical limitations make it quite difficult, costly and time consuming even though the VR system can aid in the treatment of the mental health disorders optimally.

\section{DISCUSSION AND CONCLUSION}

The virtual reality or the virtual environment has proved to be quite efficient in health care. From patient training to educating them, it helps in both the aspects. It also helps in providing training to the medical professionals in both surgical and non-surgical procedures. In surgery, the virtual device can help in remote surgery in which the surgeon is sitting at a remote location and manipulates the robotic arms which in turn performs surgery on the patient. This can help save valuable lives in the war zones and also in the rural areas where surgeons or medical professionals are not readily available. The holistic computer generated images can help the medical professionals focus on the areas that are intricate and need special attention. Also, this will help in coming up with minimally invasive surgery that will reduce blood loss in patients. In case of mental health issues such as post-traumatic stress disorders and phobias, the virtual reality systems can be used to recreate the situation that triggers the bad dreams or fears thereby making the patients immune and less reactive to their triggers. It also aids in the early detection of schizophrenia and its remedy. So this way the virtual environment also acts as a diagnostic tool. Pain reduction can also be achieved in the patients who have either undergone recent surgery or in dental treatments which are excruciatingly painful. The use of audio-visual clips helps them relax and acts as a diversion to the pain or the anticipation of pain.

The applications of virtual reality in health care and specifically $n$ mental health issues need to be spread in order to raise awareness. People at large are not aware that such a useful technology exists. Even if they are aware of it, their knowledge of the subject is largely limited to online gaming. Millions of people worldwide face social stigma due to mental disorders. The application of virtual reality in this regard will help reduce the severity. And the communication will help more and more people not only understand but also empathize with people down with any type of mental health issues.

\section{REFERENCES:}

1. Bouchard, S., Cote, S., St-Jacques, J., Robillard, G. and Renaud, P. (2006). Effectiveness of virtual reality exposure in the treatment of arachnophobia using 3D. Technol. Health Care 14(1):19-27.

2. Difede J, Hoffman H.G. (2002). Virtual Reality Exposure Therapy for World Trade Center Post-traumatic Stress Disorder: A Case Report. Cyberpsychol.Behav. 5(6):529-35

3. Freeman, D., Reeve, S., Robinson, A., Ehlers, A., Clark, D., Spanlang, B. and Slater, M. (2017). Virtual reality in the assessment, understanding, and treatment of mental health disorders. Psychological Medicine (2017), 47, 2393-2400. (C) Cambridge University Press

4. Gibson, W. (1984). Neuromancer. New York, Ace Books, 208-217

5. Haniff, D., Chamberlain, A., Moody, L. and De Freitas, S. (April 2013). Virtual environments for mental health issues: A review. Journal of Metabolomics and Systems Biology.

6. Haniff, D., Baber, C., Edmondson, W.H. (2000). Categorizing Augmented Reality Systems. J. Three Dimens. Images 14(4):105-109.

7. Hodges, L.F., Anderson, P., Burdea, G.C., Hoffman, H.G. and Rothbaum, B.O. (2001). Treating Psychological and Physical Disorders with VR. IEEE Comput. Graph. Appl. 21(6):25-33

8. Krueger, M.W. (1991). Artificial Reality ( $2^{\text {nd }}$ Edition). Reading, MA. Addison-Wesley. 208-217 
9. Lanier, J., Blanchard, C., Burgess, S., Harvill, Y., Lasko, A., Oberman, M. and Teatel, M. (1990). Reality Built For Two: A Virtual Reality Tool. ACM SIG-GRAPH Computer Graphics, Proceedings of the 1990 Symposium on Interactive $3 D$ Graphics.

10. Mandal, Sharmishtha. (April 2013). Brief Introduction of Virtual Reality and its Challenges. International Journal of Scientific \& Engineering Research, Volume 4, Issue 4

11. Mishra, P. et al. (2013). Rethinking technology and creativity in the 21st Century. TechTrends, 57(3), 10-14

12. Riva, G. (1996). Virtual Reality and Body Experience: A New Approach to the Treatment of Eating Disorders, The International Journal of Virtual Reality: A Multimedia Publication for Professionals 2, 2 (1996) 9-16 and

13. Riva, G. (February 1997). The Virtual Environment for Body-Image Modification (VEBIM): Development and Preliminary Evaluation, Presence: Tele-operators and Virtual Environments 6, 1 (February 1997) 106-117.

14. Roy, S. (2003). State of the art of virtual reality therapy (VRT) in phobic disorders. PsychNol. J. 1(2):176-183.

15. Van Dam, A., Forsberg, A., Laidlaw, D., LaViola, J. and Simpson, R. (November 2000). Immersive VR for Scientific Visualization: A Progress Report. IEEE Computer Graphics and Applications, 26-52.

16. Weimer, D. (1994). Brave New Virtual World. Frontiers of Scientific Visualization. 245-278

17. https://www.vrs.org.uk/virtual-reality-applications/

18. https://visualise.com/virtual-reality/virtual-reality-healthcare

19. https://edition.cnn.com/2017/12/13/health/virtual-reality-vr-dangers-s afety/index.html

20. https://www.who.int/en/news-room/fact-sheets/detail/mental-disorder 